First-principles electronic and elastic properties of plutonium metal

P. Soderlind, A. Landa, J. E. Klepeis

July 13, 2009

First-principles electronic and elastic properties of plutonium metal

San Francisco, CA, United States

July 12, 2009 through July 17, 2009 
This document was prepared as an account of work sponsored by an agency of the United States government. Neither the United States government nor Lawrence Livermore National Security, LLC, nor any of their employees makes any warranty, expressed or implied, or assumes any legal liability or responsibility for the accuracy, completeness, or usefulness of any information, apparatus, product, or process disclosed, or represents that its use would not infringe privately owned rights. Reference herein to any specific commercial product, process, or service by trade name, trademark, manufacturer, or otherwise does not necessarily constitute or imply its endorsement, recommendation, or favoring by the United States government or Lawrence Livermore National Security, LLC. The views and opinions of authors expressed herein do not necessarily state or reflect those of the United States government or Lawrence Livermore National Security, LLC, and shall not be used for advertising or product endorsement purposes. 


\title{
First-principles electronic and elastic properties of plutonium metal
}

\author{
Per Söderlind, A. Landa, J.E. Klepeis \\ Lawrence Livermore National Laboratory, P.O. Box 808, Livermore, CA 94550 \\ E-mail: soderlind@llnl.gov
}

\begin{abstract}
First-principles density-functional theory (DFT) calculations have been used to investigate the electronic structure and total energy of $\mathrm{Pu}$. In particular, we will present calculated electronic and elastic properties for several of its known phases. Generally, our obtained results compare favorably with the existing experimental data. Because of the limited availability of single crystals of plutonium, our calculated elastic constants are averaged to enable a comparison with data from polycrystal samples in some cases. The results presented suggest that the DFT model for plutonium is able to capture the main characteristics of the electronic structure, chemical bonding, and total energy. The magnetic properties, especially for the $\delta$ phase, remain unresolved although we show some possibilities how these could be interpreted within the framework of the presented model. Results from combining the lowtemperature first-principles calculations with a quasi-harmonic lattice-vibration model suggest that the anomalously strong temperature dependence of $\mathrm{Pu}$ chemical bonding can be largely explained without introducing additional temperature-induced electron-correlation effects.
\end{abstract}

\section{Introduction}

Plutonium is one of the least understood metals because its complex physics and chemistry are difficult to model. The electronic structure gives rise to many properties of $\mathrm{Pu}$ that are anomalous and not observed for other metals. For instance an intriguing and unusual phase diagram [1] in which atomic arrangements of sharply contrasting symmetry and density compete closely with each other. It is generally believed that these properties are related to chemical bonding but the nature of the electronic structure is not well understood on a quantummechanical level.

There have been several attempts to model the $\delta$ phase of $\mathrm{Pu}$ mainly because of the fact that electron correlation is assumed to be strong and the crystal is a simple face-centeredcubic (fcc) structure. Some of the models rely upon the density-functional theory with no or minor modifications [2,3] and others with more involved corrections [4]. The only possibility to distinguish these and other models is of course to compare with results of experimental investigations. Fortunately, there have been several recent electronic structure measurements for $\mathrm{Pu}[5,6,7]$ that can be used for this purpose. Also, Moore and van der Laan recently wrote an excellent review of the state-of-the-art situation for the actinide metals [8].

We report results from applying DFT to calculate the elastic properties of $\alpha, \beta, \gamma$, and $\delta$ plutonium. Both $\alpha-\mathrm{Pu}$ and $\beta-\mathrm{Pu}$ are monoclinic which means they have 13 independent elastic constants. The higher symmetry of the $\gamma$ phase (orthorhombic) allows for fewer independent components (9). The $\delta$ phase is, as mentioned, cubic and for this high symmetry only three 
independent coefficients survive $\left(c_{11}, c_{12}\right.$, and $\left.c_{44}\right)$. The elastic properties of a material is important for several reasons. First, they reflect details of the chemical bonding and can provide fundamental information about phase stability and strength. Second, they can be used to constrain semi-empirical models or inter-atomic potentials. Lastly, as already alluded to, they can help distinguish between proposed theoretical models.

In Sect. 2 we present some details regarding our computations. Next, the elastic properties are shown and contrasted to available experimental data in Sect. 3. We conclude in Sect. 4.

\section{Computational details}

We recently published calculated elastic constants for the $\alpha$ phase of plutonium [9] and most of the present computational details are similar and not repeated here. The more important issues are centered around the treatment of electron correlations and the electronic structure. Here, they are modeled by the generalized gradient approximation, spin polarization, and spinorbit coupling $(\mathrm{SO})$, and orbital polarization $(\mathrm{SO}+\mathrm{OP})$. This approach is the same as has been used for $\mathrm{Pu}$ in the past $[2,10]$. In the case of $\alpha$-Pu it was realized [9] that the effect of orbital polarization (OP) on the elastic constants is negligible and can safely be ignored. Here, however, we find that for $\beta-\mathrm{Pu}$ and $\gamma-\mathrm{Pu}$ the $\mathrm{OP}$ influences the elastic properties. Results for calculations including spin-orbit coupling without orbital polarization $(\mathrm{SO})$ and with $(\mathrm{SO}+\mathrm{OP})$ are therefore both presented.

An elastic modulus can be obtained from total-energy calculations of strained lattices. The details of this procedure is conventional [11] and all information was presented in our report for the $\alpha$ phase [9].

\section{Elastic constants}

Due to the very high accuracy of the calculated total energy, computations of elastic constants for more complex geometries are relatively rare, especially for actinide materials. Nevertheless, there have been such calculations presented in the literature for both $\alpha$-uranium $[12,13,14]$ and $\alpha$-plutonium [9].

We begin by showing in table 1 the results for $\alpha$-Pu taken from [9].

Table 1. $\alpha$-Pu elastic constants in GPa at $20.3 \AA^{3}$. Results for (SO) and (SO+OP) methods are nearly identical and results shown are for the former. Taken from Ref. [9].

\begin{tabular}{lllllllllllll}
\hline$c_{11}$ & $c_{22}$ & $c_{33}$ & $c_{44}$ & $c_{55}$ & $c_{66}$ & $c_{12}$ & $c_{13}$ & $c_{23}$ & $c_{15}$ & $c_{25}$ & $c_{35}$ & $c_{46}$ \\
\hline 120.0 & 108.8 & 86.2 & 43.4 & 50.6 & 43.7 & -9.30 & 1.10 & -11.5 & 22.1 & 20.2 & 21.9 & -0.25 \\
\hline
\end{tabular}

Let us first note that the $\alpha-\mathrm{Pu}$ elastic constants are here calculated without orbital polarization but at the equilibrium volume of the full theory $(\mathrm{SO}+\mathrm{OP})$. In our earlier paper [9] we reported that this approach gave very similar results to the more challanging full $(\mathrm{SO}+\mathrm{OP})$ calculations. Notice further in table 1 that some of the elastic constants are negative. This does not imply any structural instability because the strains applied to the crystal do not introduce a negative change of the total energy. For $c_{11}$ through $c_{66}$ the components are derived directly from the strains [9] while the remaining components are obtained by solving a system of linear equations defined by the geometry of the strain.

Next, we compile the results for the $\beta$ phase in table 2 . Notice here that $c_{11}$ through $c_{66}$ are significanly smaller compared to those for $\alpha$-Pu (table 1). Because they scale inversely with the atomic volume a reduction is expected when all other factors are equal. The difference in volume 
suggests a reduction of the order of $\left(\frac{20.3}{23.1}=0.88\right) 12 \%$. The actual reduction is much greater than that which is due to an overall weaker bonding in the $\beta$ phase caused by an tendency towards $5 f$-electron localization. The calculations also indicate that orbital polarization plays a more important role for the $\beta$ phase compared to the $\alpha$ phase even though the effect is not very drastic. The $(\mathrm{SO})$ calculation is performed at the equilibrium volume of the $(\mathrm{SO}+\mathrm{OP})$ treatment which partly compensates for the difference between the two approximations. For $\alpha$-Pu it completely compensated for the difference but not exactly for $\beta$-Pu. Nonetheless, the larger $\mathrm{OP}$ for $\beta-\mathrm{Pu}$, compared to $\alpha-\mathrm{Pu}$, is consistent with the fact that this phase has stronger electron correlation and localization.

Table 2. $\beta$-Pu elastic constants in GPa at $23.1 \AA^{3}$. Results for (SO) and (SO+OP) methods are shown on first and second row, respectively.

\begin{tabular}{lllllllllllll}
\hline$c_{11}$ & $c_{22}$ & $c_{33}$ & $c_{44}$ & $c_{55}$ & $c_{66}$ & $c_{12}$ & $c_{13}$ & $c_{23}$ & $c_{15}$ & $c_{25}$ & $c_{35}$ & $c_{46}$ \\
\hline 75.1 & 63.1 & 64.3 & 35.8 & 21.4 & 26.5 & 22.0 & 20.6 & 18.1 & 21.3 & 21.4 & 21.8 & -0.30 \\
75.4 & 64.4 & 65.1 & 32.2 & 22.4 & 27.2 & 27.0 & 20.6 & 23.1 & 20.6 & 21.5 & 19.4 & -0.95 \\
\hline
\end{tabular}

The succeding phase in plutonium [1] becomes stable just below $500 \mathrm{~K}$ and is the orthorhombic $\gamma-\mathrm{Pu}$. The elastic constants for this phase are collated in table 3. Here there is a greater difference between the $(\mathrm{SO})$ and the $(\mathrm{SO}+\mathrm{OP})$ theory compared to $\beta$-Pu, suggesting an increased importance of orbital polarization and even stronger $5 f$-electron correlation. The $c_{i i}$ 's, particularly for the full $(\mathrm{SO}+\mathrm{OP})$ treatment, is relatively similar to that of $\beta$-Pu but about $6 \%$ smaller on average and slightly smaller than a simple volume scaling suggests. The decrease, although small, indicates weakening of the bonding and a more correlated electronic structure in $\gamma-\mathrm{Pu}$ compared to $\beta-\mathrm{Pu}$ (and $\alpha-\mathrm{Pu}$ ).

Table 3. $\gamma$-Pu elastic constants in GPa at $23.8 \AA^{3}$. Results for (SO) and (SO+OP) methods are shown on first and second row, respectively.

\begin{tabular}{lllllllll}
\hline$c_{11}$ & $c_{22}$ & $c_{33}$ & $c_{44}$ & $c_{55}$ & $c_{66}$ & $c_{12}$ & $c_{13}$ & $c_{23}$ \\
\hline 91.1 & 67.2 & 74.5 & 28.6 & 14.1 & 27.5 & 8.80 & 22.9 & 16.0 \\
81.0 & 63.7 & 70.5 & 21.6 & 11.9 & 21.7 & 3.15 & 22.6 & 22.6 \\
\hline
\end{tabular}

The last phase we investigate is the $\delta$ phase. Here we are collecting the results published earlier [15] in table 4 together with single crystal data from Wong et al. [16]. For $\delta$-Pu there are no dramatic differences between the $(\mathrm{SO})$ and the $(\mathrm{SO}+\mathrm{OP})$ treatments when the coefficients are calculated at the $(\mathrm{SO}+\mathrm{OP})$ equilibrium volume. These calculations assume a disorered magnetic state which we have proposed to be the most relevant for this phase [17]. For comparison we also include results from a ferromagnetic (FM) calculation. The latter treatment results in a larger equilibrium volume (see below) and this likely explains the smaller $c_{44}$. The other two $\left(c_{11}\right.$ and $c_{12}$ ) do not appear to depend significantly on the magnetic configuration.

Next we summarize our equilibrium equation-of-state data in table 5 with available experimental data. We notice that the equilibrium volumes are always larger and the bulk modulus smaller when orbital polarization is included. The $(\mathrm{SO}+\mathrm{OP})$ data are also always closer to experimental data. The bulk modulus for the $(\mathrm{SO})$ treatment, evaluated at the equilibrium volume for the $(\mathrm{SO}+\mathrm{OP})$ treatment $\left(\mathrm{B}_{f i x}\right)$ is very close for $\beta, \gamma$, and $\delta$, but not for $\alpha$ plutonium. 
Table 4. $\delta$-Pu elastic constants in GPa at $24.9 \AA^{3}$, except the ferromagnetic calculation (FM) which is performed at the corresponding equilibrium volume $25.5 \AA^{3}$.

\begin{tabular}{llll}
\hline Method & $c_{11}$ & $c_{12}$ & $c_{44}$ \\
\hline SO & 62 & 28 & 43 \\
SO+OP & 65 & 29 & 48 \\
SO+OP (FM) & 63 & 29 & 27 \\
Expt & 36 & 26 & 31 \\
\hline
\end{tabular}

Table 5. Calculated and experimental equilibrium equation-of-state data. Bulk modulus (B) in GPa and atomic volume $(\mathrm{V})$ in $\AA^{3}$.

\begin{tabular}{lllll}
\hline Phase & Method & $\mathrm{V}$ & $\mathrm{B}$ & $\mathrm{B}_{\text {fix }}$ \\
\hline$\alpha-\mathrm{Pu}$ & $\mathrm{SO}$ & 19.0 & 59 & 25 \\
$\alpha-\mathrm{Pu}$ & $\mathrm{SO}+\mathrm{OP}$ & 20.3 & 50 & 50 \\
$\alpha-\mathrm{Pu}$ & Expt & $20.0-20.4$ & $40-66$ & \\
$\beta-\mathrm{Pu}$ & $\mathrm{SO}$ & 22.0 & 41 & 33 \\
$\beta-\mathrm{Pu}$ & $\mathrm{SO}+\mathrm{OP}$ & 23.1 & 37 & 37 \\
$\beta-\mathrm{Pu}$ & Expt & 22.7 & & \\
$\gamma-\mathrm{Pu}$ & $\mathrm{SO}$ & 22.7 & 38 & 30 \\
$\gamma-\mathrm{Pu}$ & $\mathrm{SO}+\mathrm{OP}$ & 23.8 & 32 & 32 \\
$\gamma-\mathrm{Pu}$ & Expt & 23.5 & & \\
$\delta-\mathrm{Pu}$ & $\mathrm{SO}$ & 24.2 & 46 & 39 \\
$\delta-\mathrm{Pu}$ & $\mathrm{SO}+\mathrm{OP}$ & 24.9 & 41 & 41 \\
$\delta-\mathrm{Pu}$ & $\mathrm{SO}+\mathrm{OP}(\mathrm{FM})$ & 25.5 & 40 & 48 \\
$\delta-\mathrm{Pu}$ & Expt & 25 & $29-30$ & \\
\hline
\end{tabular}

\section{Conclusion}

We have presented the single crystal elastic constants for $\alpha, \beta, \gamma$, and $\delta$ plutonium. For the three first phases there exist no experimental data to compare with and therefore the theoretical data serve as predictions. For the $\delta$ phase single crystal data exist and compare reasonably with experiment as we also pointed out in our previous report [15]. Through approximate averaging techniques one can attempt to relate single crystal data with that of a polycrystal. In table 6 we present Voigt averages to compare with available polycrystal data that exist for cast $\alpha-\mathrm{Pu}$ and $\delta-\mathrm{Pu}[16,18,19]$. For $\alpha-\mathrm{Pu}$ the bulk modulus appears to be somewhat underestimated whereas $G$ and $\tilde{c}_{11}$ are in very good agreement. For $\delta$-Pu the discrepancy is larger overall even though the ferromagnetic calculation is relatively close to the single crystal data. Notice that the polycrystal and single crystal data are slightly different which probably is due to errors in the averaging procedure.

Lastly, our calculations do not address thermal lattice vibrations whereas the measurements are performed at room temperature. The elastic constants show very pronounced softening with temperature [18] and it was proposed that this behavior is linked to $5 f$-electron localization. Our own investigations of $\alpha-\mathrm{Pu}$, employing Debye-Grüneisen methodology and other quasiharmonic treatments, suggest that the thermal softening of the moduli can largely be accounted for by quasi-harmonic phonon contributions with no temperature dependence of the electronic 
structure. If this is true, $5 f$-electron localization is probably not the primary driver for the thermal softening of the moduli.

Table 6. Presently calculated Voigt averages of $B, G$, and $\tilde{c}_{11}$ together with experimental data $[16,18,19]$ for cast $\alpha-\mathrm{Pu}$ and polycrystal $\delta$-Pu.

\begin{tabular}{lllll}
\hline Phase & Method & $\mathrm{B}$ & $\mathrm{G}$ & $\tilde{c}_{11}=B+4 G / 3$ \\
\hline$\alpha-\mathrm{Pu}$ & SO & 30.6 & 49.9 & 97.1 \\
$\alpha-\mathrm{Pu}$ & Expt (poly) & $46.6-54.4$ & $43.5-43.7$ & $104-112.8$ \\
$\delta$-Pu & SO+OP & 41 & 36 & 89 \\
$\delta$-Pu & SO+OP (FM) & 40 & 23 & 71 \\
$\delta$-Pu & Expt (poly) & 29.7 & 16.2 & 51.3 \\
$\delta$-Pu & Expt (single) & $29-30$ & 21 & 57 \\
\hline
\end{tabular}

\section{Acknowledgments}

This work was performed under the auspices of the U.S. Department of Energy by Lawrence Livermore National Laboratory under Contract No. DE-AC52-07NA27344

\section{References}

[1] Young D A 1991 Phase Diagrams of the Elements (University of California Press, Berkeley)

[2] Söderlind P 2001 EuroPhys. Lett 55 525; Söderlind P and Sadigh B Phys. Rev. Lett. 92185702

[3] Robert G, Pasturel A, and Siberchiot B 2003 J. Phys. Condens. Matter 15 8377; 2005 EuroPhys Lett. 71412

[4] Georges A, Kotliar G, Krauth W, Rozenberg W 1996 Rev. Mod. Phys. 6813

[5] Moore K T et al. 2007 Phys. Rev. Lett. 98236402

[6] Tobin J G et al. 2008 J. Phys.: Condens. Matter 20125204

[7] Yu S W, Tobin J G, Söderlind P 2008 J. Phys.: Condens. Matter 20422202

[8] Moore K T and van der Laan G 2009 Rev. Mod. Phys. 81235

[9] Söderlind P and Klepeis J E 2009 Phys. Rev. B 79104110

[10] Sadigh B, Söderlind P, and Wolfer W G 2003 Phys. Rev. B 68 241101(R)

[11] Wills J M, Eriksson O, Söderlind P, and Boring A M 1992 Phys. Rev. Lett. 682802

[12] Söderlind P 2002 Phys. Rev. B 66085113

[13] Taylor C D 2008 Phys. Rev. B 77094119

[14] Bouchet J 2008 Phys. Rev. B 77024113

[15] Söderlind P, Landa A, Sadigh B, Vitos L, and Ruban A 2004 Phys. Rev. B 70144103

[16] Wong J et al. 2003 Science 3011078

[17] Söderlind P, Landa A, Sadigh B 2002 Phys. Rev. B 66205109

[18] Migliori A, Mihut I, Betts J B, Ramos M, Mielke C, Pantea C, and Miller D 2007 J. Alloys Compd. 444-445 133

[19] Wick O J 1967 Plutonium Handbook A Guide to the Technology (Gordon and Breach, New York) 